\title{
A State-of-the-Art Review on Segmentation Algorithms in Intravascular Ultrasound (IVUS) Images
}

\author{
Amin Katouzian, Elsa D. Angelini, Member, IEEE, Stéphane G. Carlier, Jasjit S. Suri, Nassir Navab, \\ and Andrew F. Laine, Fellow, IEEE
}

\begin{abstract}
Over the past two decades, intravascular ultrasound (IVUS) image segmentation has remained a challenge for researchers while the use of this imaging modality is rapidly growing in catheterization procedures and in research studies. IVUS provides cross-sectional grayscale images of the arterial wall and the extent of atherosclerotic plaques with high spatial resolution in real time. In this paper, we review recently developed image processing methods for the detection of media-adventitia and luminal borders in IVUS images acquired with different transducers operating at frequencies ranging from 20 to $45 \mathrm{MHz}$. We discuss methodological challenges, lack of diversity in reported datasets, and weaknesses of quantification metrics that make IVUS segmentation still an open problem despite all efforts. In conclusion, we call for a common reference database, validation metrics, and ground-truth definition with which new and existing algorithms could be benchmarked.
\end{abstract}

Index Terms-Intravascular ultrasound (IVUS), lumen, mediaadventitia (MA), segmentation.

\section{INTRODUCTION}

\section{A. Medical Background}

F OR more than 30 years after its introduction by Andreas Grüntzig in 1977, percutaneous coronary interventions (PCI) remain the most widely used methods by interventional cardiologists to treat coronary artery disease. Lumenology was initially used for guidance of the interventions based on Xray angiography [1], which had been accidently discovered by Sones and later deployed for coronary catheterization [2]. Invention and refinement of intravascular ultrasound (IVUS) imaging has introduced in vivo "histological" assessment of coronary atherosclerosis and plaques. As an alternative, invasive coronary angiography depicts planar projections of the contrast-filled lumen. Important quantitative information such as cross-sectional

Manuscript received September 30, 2011; revised January 18, 2012; accepted February 19, 2012. Date of publication February 28, 2012; date of current version September 20, 2012

A. Katouzian is with the Technical University of Munich, Munich 80333, Germany, and also with Heffner Biomedical Engineering Imaging Laboratory, Columbia University, NY 10027 USA (e-mail: amin.katouzian@cs.tum.edu).

E. D. Angelini is with the Institut Telecom, Telecom ParisTech, CNRS LTCI Paris 75013, France (e-mail: elsa.angelini@ telecom-paristech.fr).

S. G. Carlier is with the Department of Cardiology, Universitair Ziekenhuis, Brussel 1090, Belgium (e-mail: sgcarlier@ hotmail.com).

J. S. Suri is with Global Biomedical Technologies, Inc., CA 95661 USA, and also with Idaho State University, ID 83209 USA (e-mail: jsuri@ comcast.net).

N. Navab is with the Technical University of Munich, Munich 80333, Germany (e-mail: navab@cs.tum.edu).

A. F. Laine is with the Departments of Biomedical and Radiology, Columbia University, New York, NY 10027 USA (e-mail: laine@ columbia.edu).

Color versions of one or more of the figures in this paper are available online at http://ieeexplore.ieee.org.

Digital Object Identifier 10.1109/TITB.2012.2189408 lumen area, thickness, distribution, and composition of plaques and remodeling of the vessel wall are only revealed by IVUS that can improve and guide PCI. Moreover, it enables the monitoring of regression and progression of plaques by measuring changes over time of the atheroma volume within the vessel wall, especially when evaluating new pharmacological compounds. Besides, for chronic disease such as atherosclerosis that may reoccur after balloon angioplasty, atherectomy, stenting, or bypass surgery, the accurate diagnosis of vulnerable plaques [3]-[7] is critical. In brief, what makes atherosclerosis one of the deadliest disease is not the stenoses alone but failure in detection and proper treatment of the vulnerable plaques that will lead to myocardial infarction. This point has motivated researchers to develop novel imaging modalities such as IVUS, optical coherence tomography (OCT) [8], or near-infrared signals (NIR) [9] to characterize atherosclerotic plaque components and identify vulnerable ones.

\section{B. Specificities of IVUS Images}

Angiography is the only system routinely used in all PCI procedures. It provides immediate visualization of stenoses and guides interventional cardiologists to advance and deploy balloons and stents. However, it suffers from the lack of adequate geometrical and pathological information on plaque burden size and composition. So far, IVUS remains the most favorable imaging modality for coronary plaques for the following reasons.

1) It provides real-time cross-sectional grayscale images of the arterial wall, including morphological and pathological structures. Image resolution and signal penetration are sufficient to allow precise tomographic assessment of the coronaries.

2) IVUS grayscale images combined with the processing of radiofrequency backscattered signals can be employed for further characterization of plaques and the identification of vulnerable ones [10].

3) Interventional cardiologists can make therapeutic decisions from IVUS images, such as:

a) the need for further treatment (angioplasty, stent implantation, and bypass);

b) the exact spatial location for angioplasty and stent implantation;

c) the evaluation of the outcome of an angioplasty or stenting procedure;

d) the need for aggressive management of risk factors prior to onset of symptoms and advanced disease state;

e) the predictors of transplant coronary artery disease. 


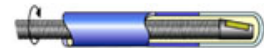

(a)

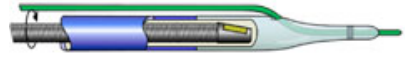

(b)

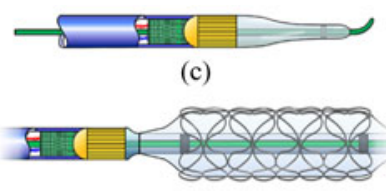

(d)
Fig. 1. Schematic of four different types of imaging catheters. (a) Catheter without guide wire rail. (b) Catheter with a guide wire rail on the side. (c) Catheter with a guide wire rail at its center. (d) Catheter with a guide wire rail at its center, an inflatable balloon, and a stent. Reference: http://ee.isikun.edu.tr/research.asp-page = projects_files.

Comparing with other modalities, the scientific and diagnostic advantages of IVUS are clear. The American College of Cardiology and the American Heart Association (ACC/AHA) guidelines for PCI state that "the limitations of coronary angiography for diagnosis and interventional procedures can be reduced by the use of adjunctive technology such as intracoronary ultrasound imaging" and that IVUS can improve PCI methods and outcomes [11]. Additionally, IVUS is the primary screening choice for validation of novel endovascular coronary imaging modalities (i.e., OCT and NIR). It has been widely used in prospective trials to investigate the efficacy of new endovascular devices or drugs. With respect to the detection of vulnerable plaques, IVUS could become the most common and trustworthy screening technique if reliable and reproducible image or signal processing methods are provided for quantitative plaque characterization. Regarding vulnerable plaques, IVUS could encompass a greater predictive value in detecting them with the combination of morphological features (thin-cap fibro atheroma (TCFA), lipid core size, and calcification patterns) that are all detectable and measurable at once, which is not the case for competitive imaging modalities such as NIR and OCT. Among the morphological features, TCFA $(<65 \mu \mathrm{m})$ is better depicted and measured on OCT images with a higher resolution $(\sim 10$ $\mu \mathrm{m})$ comparing to IVUS $(\sim 120 \mu \mathrm{m})$. This limitation may be resolved in the future, developing ultrahigh-frequency IVUS transducers that provide images with higher axial resolutions.

\section{IVUS Acquisition Systems}

The IVUS acquisition system consists of a catheter, a pullback device, and a scanning console.

1) IVUS Catheter: The IVUS catheter carries an ultrasound transducer that can be combined with an inflatable balloon, with or without a stent, for imaging assistance and expansion of narrowed areas, as illustrated in Fig. 1. The IVUS catheter is $150 \mathrm{~cm}$ long, and has a tip size of $3.2-3.5 \mathrm{~F}(1.2-1.5 \mathrm{~mm})$ that can go through $5-6 \mathrm{~F}$ guiding catheter. It may be used to visualize over $15 \mathrm{~cm}$ of a coronary artery. The imaging field goes up to $15-20 \mathrm{~mm}$, well enough for coronary arteries, ranging from 4 to $5 \mathrm{~mm}$ in diameter on average at the level of the left main artery down to $2 \mathrm{~mm}$ in the smallest segment considered for therapeutic intervention (balloon angioplasty and stenting). The catheter is typically advanced within the femoral artery toward coronary arteries and site of occlusion under angiogram guidance. The catheter is visible in angiographic images and is advanced along with a guide wire. The guide wire rail is positioned next to the catheter plastic sheath, as in Fig. 1(b),

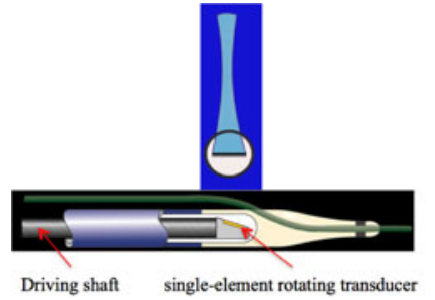

(a)

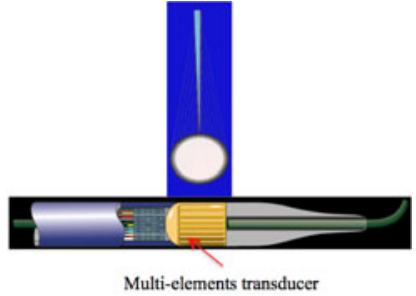

(b)
Fig. 2. (a) Single-element mechanically rotating focused IVUS transducer and its beam shape. (b) Multi-element phased-array IVUS transducer and its beam shape.

or within its center, as in Fig. 1(c). The advantage of the latter design is that there is no guide wire artifact in the reconstructed grayscale ultrasound images, but at the cost of usually stiffer, thicker, and less flexible catheters.

2) IVUS Transducer: Currently, there are two types of IVUS transducers commercially available regardless of their nominal center frequencies. The main difference relies in the transmit and receive modes for monitoring ultrasound signals, which are illustrated in Fig. 2. The first system, illustrated in Fig. 2(a), uses a single-element mechanically rotating focused IVUS transducer (e.g., Atlantis, Boston Scientific imaging catheter) that rotates at approximately 1800 revolutions/min. For a $40-\mathrm{MHz}$ transducer, the axial and lateral resolutions of the beam are about 80-100 and 200-250 $\mu \mathrm{m}$, respectively. The transducer sends an ultrasound pulse and receives the backscattered signals. The transducer is surrounded by a plastic sheath and a syringe is used to flush saline water inside the sheath to remove air bubbles and obtain high-quality IVUS images.

The second system, illustrated in Fig. 2(b), uses a multielement phased-array transducer (e.g., Eagle Eye Gold, Volcano imaging catheter). An electronic board controls a subset of elements to send several ultrasound pulses at once and receive the backscattered signals. These circular array systems use synthetic aperture processing to produce images with higher lateral resolution than single-element transducers.

3) Catheter Pullback Device: The catheter is first manually advanced to the distal end of the coronary (typically after the stenoses location) and is then pulled back, manually or with an automatic pullback system, at a speed of $0.5-1 \mathrm{~mm} / \mathrm{s}$.

4) IVUS Scanning Consoles: A scanning console carries a computer that is used for postprocessing and storage of recorded IVUS data. A cable from the end of the pullback device is connected, via a dedicated port, with a computer for data processing. During the catheterization procedure, the clinician uses a keyboard and functional buttons to enter the patient information, determine the percentage of stenoses, and apply image processing and possibly tissue characterization techniques to better understand and evaluate atherosclerotic plaques.

\section{IVUS Image Formation and Display}

IVUS transducers operate at different frequencies, depending on the manufacturer. Fig. 3 displays the schematic of an artery, an IVUS catheter, and four distinct IVUS image frames acquired with transducers with different center frequencies. As 


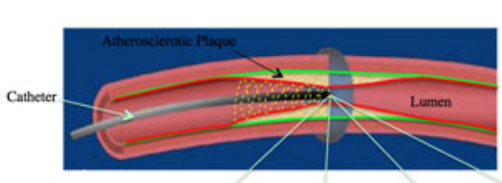

(a)

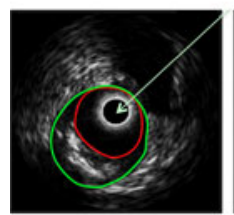

(c)

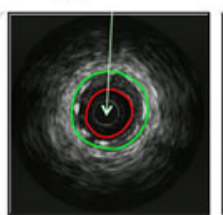

(d)

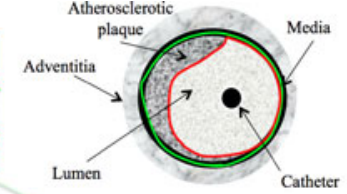

(b)

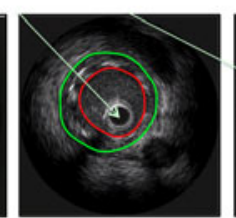

(e)

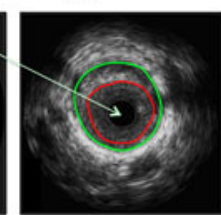

(f)
Fig. 3. (a) Schematic of an artery, catheter, atherosclerotic plaque, and IVUS image cross section (reference: http://www.bmj.com). (b) Cross-sectional anatomy of the arterial wall. Four distinct IVUS frames acquired with (c) 20-MHz, (d) 30-MHz, (e) 40-MHz, and (f) 45-MHz transducers. Green and red borders represent the vessel wall (MA) and lumen (intima) borders, respectively. The yellow dashed line depicts the trajectory of transducer scan lines.

illustrated, at higher center frequency, spatial resolution is improved, at the cost of more scattering from red blood cells inside the lumen. It is worth mentioning that the axial and lateral resolutions depend on the transducer center frequency and beam width, respectively.

During acquisition, IVUS backscattered radiofrequency (RF) signals that are continuous-time real-valued and band-limited signals, $x(t)$, are digitized $x\left(n T_{s}\right)=x_{n}$ at periodic time intervals of $T_{s}=f_{s}^{-1}$ and stored in the hard disk of a computer. $f_{s}$ is the sampling rate of the digitizing board and may vary from one system to another. For example, in the Boston Scientific (Fremont, CA) Galaxy or iLab imaging systems, the acquisition boards sample IVUS signals at the rate of $f_{s}=400 \mathrm{MHz}$ whereas the sampling rate for the Volcano $s 5^{\mathrm{TM}}$ imaging system is $f_{s}=200 \mathrm{MHz}$. Once the IVUS backscattered signals are digitized, numbers of steps need to be taken in order to convert digitized RF signals into typical IVUS grayscale images. First, the envelope of each RF signal (A-line) is computed to generate a corresponding analytical signal [12]. This is followed by decimation and interpolation along the axial and lateral directions, respectively. Log compression is also used to enhance image quality followed by a quantization (e.g., 8 bit).

As depicted in Fig. 3(a), the transducer has a spiral trajectory (yellow dashed line) while acquiring cross-sectional grayscale images. The original domain of acquisition is polar $(r, \theta)$ and the resulting grayscale image is transformed to $(x, y)$ Cartesian coordinates to reconstruct a typical IVUS frame. Planar cuts through stack of cross-sectional images provide longitudinal views of an artery. Interventional cardiologists can assess the length of an artery and the distribution of atherosclerotic plaques within this pullback direction. Fig. 4 illustrates an IVUS grayscale image in polar and Cartesian coordinates along with an example of a cut in the longitudinal pullback direction.

\section{E. IVUS Image Artifacts}

IVUS images may suffer from severe acquisition artifacts. We can cite five main artifacts: presence of the guide wire,

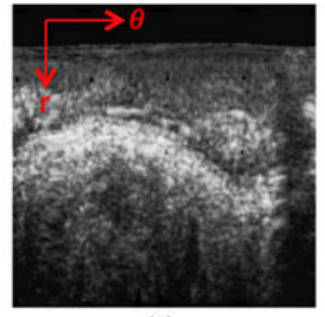

(a)

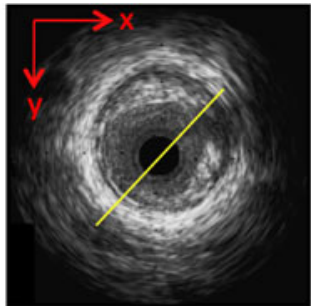

(b)

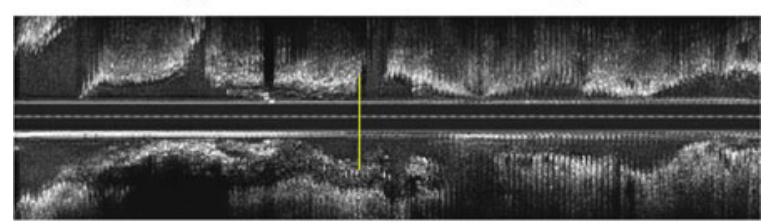

(c)

Fig. 4. IVUS grayscale image in (a) polar $(r, \theta)$ and (b) $(x, y)$ Cartesian domains. (c) Longitudinal display along an arbitrary planar cut identified as the yellow line in (b).

ring-down, nonuniform rotational distortion (NURD), reverberation, and discontinuity at $0^{\circ}$ in the Cartesian domain. When a guide wire rail is designed along with a plastic sheath of the catheter, it obstructs the propagation of ultrasound signals, resulting in shadowing behind the guide wire, as illustrated in Fig. 5(a). The second artifact arises from the repetitive reflections of the ultrasound signal from the surface of the transducer because of impedance mismatch, as illustrated in Fig. 5(b). The NURD artifact is due to a mechanical glitch in the driving shaft or the binding of the catheter in curved arteries, as illustrated in Fig. 5(d). The fourth artifact, known as reverberation, corresponds to oscillations of the ultrasound signals between the transducers and the arc of calcified plaques, which causes multiple appearances of calcified arcs, as illustrated in Fig. 5(c). The last artifact corresponds to a discontinuity of tissue appearance at $0^{\circ}$ in the Cartesian domain due to the spiral trajectory of the transducer as well as severe catheter or heart motions, as illustrated in Fig. 5(a).

\section{F. In Vivo Data Collection}

Generally, an IVUS catheter is advanced into the left or right coronary artery and possibly in some side branches on a guide wire coming out of a guiding catheter inserted in the femoral artery. Acquisition of cross-sectional ultrasound images of the right coronary arteries (RCA), left anterior descending (LAD), and left circumflex (LCX) coronary arteries can be performed with a rotating single-element transducer or a phasedarray transducer. The catheter pullback speed varies between 0.5 and $1 \mathrm{~mm} / \mathrm{s}$ and the frame rate can be set to 30-60 frames/s. The IVUS RF data and images are acquired as described in Section I-D.

\section{G. Image Processing Challenges}

During a catheterization procedure, hundreds to thousands of IVUS images are recorded. Therefore, automatic detection of the vessel wall [media-adventitia (MA)] and luminal borders is required to quantify the degree of stenoses and measure 


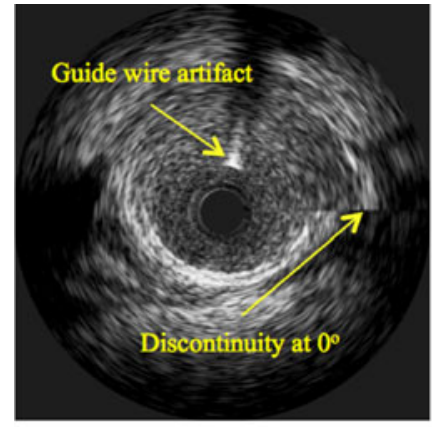

(a)

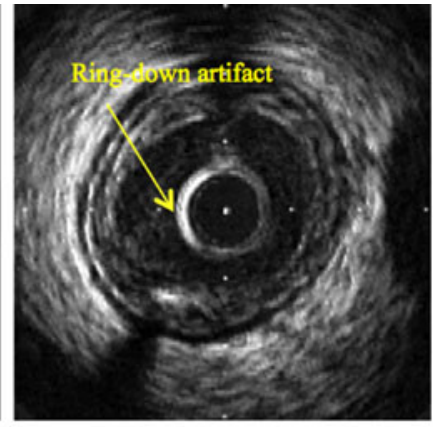

(b)

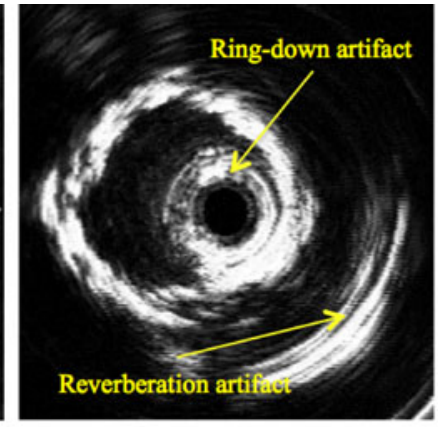

(c)

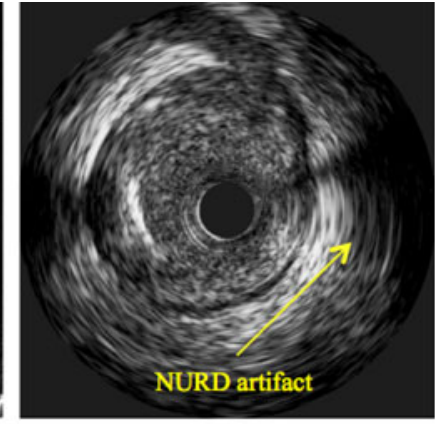

(d)

Fig. 5. IVUS image artifacts. (a) Guide wire artifact and discontinuity artifact at $0^{\circ}$. (b) Ring-down artifact. (c) Ring-down artifact and reverberation artifact. (d) NURD artifact. Calibration markers (small white squares) in (b) are used for measurements.

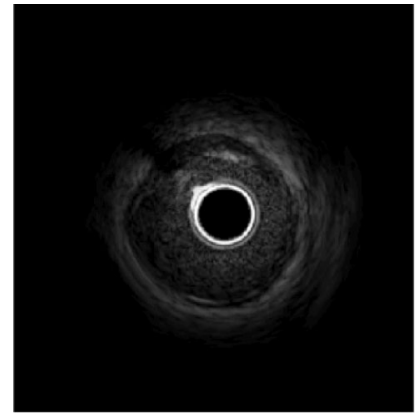

(a)

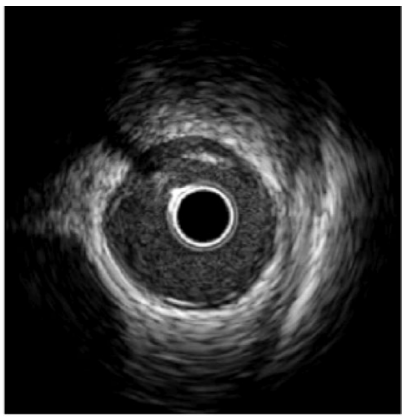

(b)

Fig. 6. IVUS grayscale image (a) without TGC adjustment and (b) with TGC adjustment.

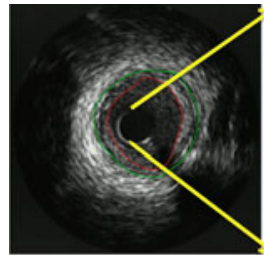

(a)

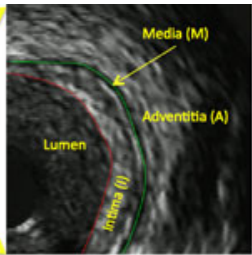

(b)

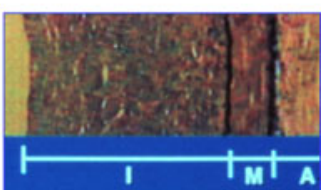

(c)
Fig. 7. IVUS grayscale image with (a) manually traced vessel wall (green) and lumen (red) borders, (b) zoomed-in region with anatomical structures of the artery such as lumen, intima (I) or plaque, media (M), and adventitia (A), (c) histology image of artery anatomical structures.

the luminal area in which blood flows. The lumen border is at the innermost surface of atherosclerotic plaques. Since ultrasound signals are progressively weakened with depth, time gain compensation (TGC) can be applied to compensate for this, as illustrated in Fig. 6. The vessel wall border, also called the external elastic membrane (EEM) border, is a contour drawn at the interface between the media and the adventitia. Made of smooth muscle cells, the media does not reflect the ultrasound signal and appears as a dark ring. Adventitia is the outer layer of an artery, formed of sheets layers that are hyperechogenic and appear as a bright region. Fig. 7 illustrates the borders, the corresponding anatomical structures in an IVUS grayscale image and a histology image of an artery.

Generally speaking, detection of vessel wall borders is less difficult than that of lumen borders since the vessel media con- sists of smooth muscle cells and does not reflect ultrasound signals. It appears as a dark region on IVUS images, which can be used as a marker to detect the vessel wall. In contrast, due to high scattering from red blood cells inside the lumen, the detection of the luminal border is more challenging, especially when a high-frequency transducer is used. Comparing IVUS ultrasound probes, the lumen border is more easily detected in images acquired with a 64-element phased-array $20-\mathrm{MHz}$ transducer than with a single-element mechanically rotating $45-\mathrm{MHz}$ transducer, as illustrated in Fig. 3.

In any case, clinical applications of automated segmentation methods have seen limited success due to several intrinsic artifacts (presence of the guide wire, presence of calcified plaques, presence of side branches, motion of the catheter and the heart) and extrinsic parameters (such as manual setting of TGC). For example, the presence of the guide wire, calcified plaques, and side branches significantly affects an algorithm performance, particularly when deformable models are employed. On the other hand, variability among system specifications or changes of acquisition parameters by an expert would lead to inconsistency among datasets so that supervised techniques or those that rely on statistical properties of gray level intensities may not perform efficiently.

In this paper, we review state-of-the-art segmentation algorithms that detect either both borders simultaneously or one of them. These methods can be categorized based on their clinical application, domain of analysis, transducer center frequency, dimensionality, and underlying image processing framework, as summarized in Table I. To tackle the segmentation problem, researchers have developed several algorithms, employing different techniques such as graph searching, gradient-driven methods with dynamic programming, deformable models in combination with statistical properties of grayscale values corresponding to blood and nonblood regions, statistical shape models, probabilistic approaches, edge-enhancement frameworks along with active contours, or multiscale techniques, for example. We distinguished three main families of approaches: 1) direct detection of border(s); 2) blood speckle reduction (i.e., spatiotemporal filtering) as a preprocessing step prior to border detection; and 3) supervised classification [e.g., support vector machine (SVM)] of blood versus nonblood regions by extracting appropriate spatial/temporal/spectral features. For each family of methods, we describe the main principles, performance, 
TABLE I

List of Some of EXISTING SEgmentation ALgorithms In IVUS IMAgES AND THEIR SPECIFICATIONS

\begin{tabular}{|c|c|c|c|c|c|c|}
\hline Authors [Ref. \#], Year & $\begin{array}{c}\text { Implemented } \\
\text { Technique }\end{array}$ & Application & $\begin{array}{c}\text { Domain of } \\
\text { Analysis }\end{array}$ & $\begin{array}{c}\text { Transducer } \\
\text { Freq. (MHz) }\end{array}$ & Dataset & $\begin{array}{l}\text { Validation } \\
\text { Technique }\end{array}$ \\
\hline $\begin{array}{c}\text { Sonka et al. }[14,17] \\
1995,2008\end{array}$ & 2D, Graph search & MABD \& LuBD & Polar & 30 & 38 frames from 24 hearts, in vitro & LRA, $_{\text {RMSE }}$ min,max \\
\hline $\begin{array}{c}\text { Birgelen } \text { et al. [18], } \\
1996\end{array}$ & 3D, Dynamic programming & MABD \& LuBD & $\begin{array}{c}\text { Transversal and } \\
\text { longitudinal }\end{array}$ & 30 & $\begin{array}{l}\text { Phantom data, } 200 \text { frames from } 20 \\
\text { in vivo cases }\end{array}$ & LRA, BAA, IIC \\
\hline Meier et al. [23], 1997 & $\begin{array}{l}\text { 2D, Spatial filtering, region } \\
\text { growing, and snake }\end{array}$ & MABD \& LuBD & Polar & 30 & $\begin{array}{l}105 \text { frames ( } 77 \text { for LuBD and } 28 \text { for } \\
\text { MABD) }\end{array}$ & LRA, BAA, IIC \\
\hline $\begin{array}{c}\text { Shekhar et al. [37], } \\
1999\end{array}$ & 3D, Deformable model & MABD \& LuBD & Cartesian & 30 & $\begin{array}{l}78 \text { in vivo from } 8 \text { hearts for LB, } 83 \\
\text { in vitro frames ( } 55 \text { for LuBD, } 28 \text { for } \\
\text { MABD) }\end{array}$ & $\begin{array}{l}\text { LRA, } \mathrm{WI}_{\text {Area }}, \mathrm{WI}_{\text {Perimeter, }}, \\
\mathrm{WI}_{\mathrm{AD}}, \mathrm{WI}_{\mathrm{HD}}\end{array}$ \\
\hline $\begin{array}{l}\text { Kovalski et al. [35], } \\
2000\end{array}$ & 3D, Deformable model & MABD \& LuBD & Polar & 30 & 88 gated frames from 8 in vivo cases & LRA, IIC \\
\hline $\begin{array}{l}\text { Brusseau et al. [42], } \\
2004\end{array}$ & 2D, Probabilistic Approach & LuBD & Polar & 30 & 15 frames ${ }^{*}$ from 15 in vivo cases & $\begin{array}{c}\text { LRA, } \\
\text { Error }_{\text {mean, variance, } \min , \max }\end{array}$ \\
\hline $\begin{array}{l}\text { Cardinal } \text { et al. [57], } \\
2006\end{array}$ & 3D, PDF-based fast marching & MABD \& LuBD & Polar & 20 & 600 frames from 9 in vivo cases & $\begin{array}{l}\text { AD, HD, Area } \\
\text { Correlation Coeff. }\end{array}$ \\
\hline Gil et al. [64], 2006 & $\begin{array}{c}\text { 2D, Supervised Classification, } \\
\text { Snake }\end{array}$ & MABD & Polar & 40 & 5400 frames from 11 in vivo cases & $\begin{array}{l}\text { MaxD, RMaxD, MD, } \\
\text { RMD, MSD }\end{array}$ \\
\hline Taki et al. [49], 2008 & $\begin{array}{l}\text { 2D, Statistical Analysis, Active } \\
\text { contour }\end{array}$ & MABD \& LuBD & Cartesian & 30 & 60 in vivo frames from 7 cases & LRA, AD, HD \\
\hline Unal et al. [46], 2008 & 2D, Statistical shape model & MABD \& LuBD & Polar & 20 & 1919 in vivo frames & $\begin{array}{c}\text { FPR, FNR, OR, } \\
\text { Distance }_{\text {min,max }}, \text { BAA, }\end{array}$ \\
\hline $\begin{array}{c}\text { Katouzian } \text { et al. } \\
{[63,74], 2011,2010}\end{array}$ & 3D, Multiscale & LuBD & Polar & $40 \& 45$ & $\begin{array}{c}\text { In vivo, } 1158 \text { frames from } 5 \text { cases } \\
(45 \mathrm{MHz}), 320 \text { frames from } 7 \text { cases } \\
(40 \mathrm{MHz})\end{array}$ & $\begin{array}{l}\text { LRA, BAA, TP, FP, } \\
\text { RMSE }\end{array}$ \\
\hline Hibi et al. [84], 2000 & 3D, Spatio-Temporal Analysis & BNR & Polar & 40 & 682 in vivo frames from 35 cases & LRA, IIC \\
\hline $\begin{array}{l}\text { Balocco et al. }[80] \\
2003\end{array}$ & 3D, Spatio-Temporal Analysis & BNR & Cartesian & 30 & $\begin{array}{l}4 \text { simulated frames, unknown } \\
\text { number of in vitro frames }\end{array}$ & PFOM, HD, MADE \\
\hline $\begin{array}{l}\text { Katouzian et al. [73], } \\
2009\end{array}$ & 3D, Multiscale & BNR & Polar & 45 & 233 in vivo frames from 3 cases & LRA, Tanimoto $_{\min , \max }$ \\
\hline Rotger et al. [86], 2007 & Adaboost classifier & BPD & Longitudinal & $\begin{array}{l}\text { Not reported } \\
\text { (looks 40) }\end{array}$ & Not reported & Not reported \\
\hline $\begin{array}{l}\text { O'Malley et al. [81], } \\
2007\end{array}$ & $\begin{array}{l}\text { 3D, Spatio-tempral-spectral } \\
\text { Analysis }\end{array}$ & BPD & Polar & 40 & 90 in vivo frames from 3 cases & TP, FP, TN, FN, SE, SP \\
\hline $\begin{array}{l}\text { Katouzian et al. [82], } \\
2009\end{array}$ & 3D, Multiscale & BPD, LuBD & Polar & 45 & 258 in vivo frames from 3 cases & $\begin{array}{c}\text { FP, FN, SE, SP, } \\
\text { Tanimoto }_{\text {mean,min,max,SD }}\end{array}$ \\
\hline
\end{tabular}

advantages, and limitations. Particular attention was also paid to the level of automation of each technique. Some methodologies, lack complete automation and require user interaction in order to perform the segmentation tasks.

\section{Direct Detection OF BORDER(S)}

\section{A. Edge-Tracking and Gradient-Based Techniques}

The interactions of IVUS signal with the blood-tissue interface and smooth muscle cells in media give rise to typical edge patterns that could be used to distinguish lumen and MA contours, respectively. In practice, these patterns seldom embody clean borders due to scattering effects within the lumen, discontinuity in intensity values, drops in edge reflections, noise. Hence, further refinement (e.g., smoothing for noise reduction) and hybrid algorithms were designed to assemble edge features into desirable target boundaries. The IVUS segmentation techniques that deploy such image descriptors usually require precise initialization and rely on an energy minimization framework. The very first work on IVUS border detection from Herrington et al. [13] developed a semiautomated algorithm based on such principle. Later, Sonka et al. [14] introduced one of the earliest comprehensive works on the detection of internal and external elastic laminae borders as well as lumen borders. The internal and external elastic laminae borders refer to the inner and outer layers of the media, which consist of smooth muscle cells. Normally, the MA border can be drawn anywhere between these two borders (within the corresponding hypoechoic region). After removing the calibration markers, illustrated in
Fig. 5(b), regions of interest (ROIs) were interactively selected and Sobel-like edge detectors were applied on subimages to construct laminae and lumen border graphs. A heuristic graph search technique [15], [16] was then performed deploying two distinct cost functions to detect the borders. The key point for precise identification of borders was to define appropriate cost functions for each border by incorporating a priori knowledge such as shape models and edge patterns. The results demonstrated good correlation between manual and automated lumen borders $(r=0.96)$, plaques $(r=0.95)$, and stenoses areas $(r$ $=0.93$ ). Although the presented technique required some user interaction and was only applied on in vitro images using circulating saline water, where there was not much scattering in the lumen area compared to in vivo images, the results were encouraging and this study raised attentions toward this particular problem. An extended version of this approach using a different cost function and fully 3-D graph search has been presented in [17].

With a similar type of approach, the authors in [18] and [19] presented a semi-automated methodology using dynamic programming to find the optimal path within the vessel and detect both MA and lumen borders in polar coordinates from delineated contours. The minimal path search was performed between end points interactively selected in reconstructed longitudinal images at the intersection of two perpendicular cut planes. The results were validated using both tubular phantom data and in vivo images acquired with a $30-\mathrm{MHz}$ transducer [18]. They studied inter- and intraobserver variability and showed high consistency of the method. They later evaluated the performance 
of the segmentation by comparing to histological images [20]. The algorithm performance was further refined and correlation between manual and automated traced contours was improved from $(r=0.91)$ to $(r=0.98)$ by employing electrocardiogram (ECG)-gated images [21]. The main limitations were related to non-uniform transducer rotation and high curvature of the arterial vessel shape that created distortions in planar images. In addition, accurate positioning of an individual transverse plane in longitudinal sections was crucial and could affect the quality of the segmentation. A similar semi-automated knowledgeguided approach was also proposed by the authors in [22].

Meier et al. [23] proposed a fully automated segmentation method for the detection of both MA and lumen borders through the enhancement of image continuity along the circumferential direction in polar coordinates and speckle noise reduction by applying iterative nonlinear spatial median filters. Three different segmentation techniques were applied to detect the lumen borders: 1) thresholding of grayscale gradient maps obtained by convolving the polar image with gradient kernels in the radial direction; 2) adaptive region growing from a luminal seed point (after the detection of the catheter); and 3) deformation of a gradient-based parametric deformable model to search for connected outline points and detect the MA border. Postprocessing was required to remove remaining outliers and refine the final segmentation. Two distinct datasets consisting of 77 and 28 in vivo collected IVUS frames acquired with a single-element $30-\mathrm{MHz}$ transducer were then used for the detection of lumen and MA borders, respectively. The authors in [24] proposed a modified image cost function, combining gradient and variance of grayscale intensities, which was less sensitive to noise and employed circular dynamic programming for the detection of the MA borders. The presented algorithm required manual initialization of ROI in the first frame of analysis. Analogous to the method in [23], Schmauder et al. [25] used dedicated preprocessing (i.e., median filtering and histogram equalization) to remove catheter markers and scaling grids. They started with an initialized contour to confine the segmentation process within ROI. A radial search procedure was performed, optimizing an energy functional formulated as a weighted linear combination of an edge detector (first derivative of a Gaussian operator), an appearance feature (mean value of intensity measured within sliding windows), and a smoothing term. A multi-temperature simulated annealing optimization was then used to minimize the energy functional and detect the lumen border. The performance of the algorithm was evaluated using 160 frames collected from five patients using a single-element $30-\mathrm{MHz}$ transducer.

\section{B. Active Contour-Based Techniques}

Active contours have been widely used in many medical image segmentation applications and most of the IVUS studies adopted the traditional formulation of a parametric snake [26], [27]. Rather than implicit shape surface representation to retrieve lumen and/or MA boundaries. Parametric model formulation is more intuitive and better IVUS border detection applications since the topology of different boundaries is simple and underlying parameterization remains simple and computationally efficient. The tradeoff of using parametric representations relies in the fact that it is more sensible to initial conditions because of nonconvexity of the energy functional and the need for advection forces. Due to intrinsic non-vessel image features (presence of guide wire, calcified plaques, side branches, motion artifacts from the catheter and the heart) and image variability due to extrinsic parameters (system parameter specifications such as TGC and compression of the dynamic range), the vessel borders are not well distinguished in IVUS image which hinders the direct use of a classical deformable model. Moreover, finetuning of parameters and proper initialization are required when ordinary features such as image gradient and intensity, which are sensitive to noise, are employed. Hence, several approaches have been proposed to overcome these drawbacks by modifying the energy terms or incorporating pre-processing techniques prior to the use of a deformable model. For example, the authors in [28] and [29] applied nonlinear filters [30]-[32], driven from the heat diffusion process, to homogenize grayscale values and enhance edges prior to the detection of MA and lumen borders, respectively, through a classical snake framework [26]. The authors in [33] utilized a Hopfield neural network [34] to solve the optimization problem the snake framework with lesser computational complexity. In addition, they modified the definition of the bias of neurons, incorporating image characteristics as $a$ priori, to overcome possible distortions in textural patterns due to the presence of arcs of calcified plaques and detected both borders. The user only needed to initialized lumen and MA borders in the very first frames and resulting detected borders then were automatically employed as initial contours for subsequent frames.

As an alternative automated approach, Kovalski et al. [35] removed the elasticity term [26] from the internal energy. To control the smoothness of the contour, they introduced an a priori on the final desired shape via regularization along longitudinal direction, and a "balloon" force [36] to control point motion only along radial directions. The contours evolved toward features of interest driven by intensity-based external forces. Finally, they extended the algorithm to 3-D for the automatic detection of MA and lumen borders. The results demonstrated high correlation $(r=0.978)$ and low variability $15.2 \pm 17.4 \%$ and $6.5 \pm 7.6 \%$ for lumen and MA borders, respectively, when comparing to manual tracing. The authors in [37] also developed a semiautomated 3-D segmentation framework called the active surface model, which was an extension of a 2-D digital dynamic model (DDM) technique [38], for the detection of both borders. The internal force comprised distinct tangential and radial components derived from corresponding transverse and longitudinal edge vectors. The external force was based on the gradient of a 3-D potential field defined by convolving the image volume with a 3-D Sobel-like kernel. A damping force was used to control the convergence of the model to its final shape. The algorithm required user intervention to initialize the template every other ten frames. The results demonstrated robust segmentation with negligible variability. Later, in a comprehensive study [39], the performance of the algorithm was evaluated against more computationally efficient 3 -D active surface algorithm using a so-called neighborhood-search model.

The proposed technique in [37] is of special interest since it demonstrated encouraging results and potentially can be used 
images acquired with transducers. Although the lumen and MA borders had been detected in two distinct datasets (see Table I), simultaneous detection of both borders seems feasible.

\section{Statistical-and Probabilistic-Based Techniques}

Statistically driven approaches are generally established upon an assumption that grayscale values corresponding to lumen and plaque (intima) regions are generated from two different distributions of reflectors and could be modeled parametrically [e.g., Rayleigh or mixture of Gaussians probability density functions (pdf)] [40]-[45] or non-parametrically [46]. In many cases, this is a valid assumption to differentiate blood textures (speckles) from tissues and detect the lumen borders. The authors in [47] took advantage of this property and developed the very first region-based technique for the detection of lumen borders by hard-thresholding of images in polar coordinates. They incorporated probabilities of grayscale values that corresponded to lumen and non-lumen areas into a deformable model framework, generated corresponding edge images, and delineated borders automatically in Cartesian coordinates with elliptical shape constraint. The algorithm was tested on 1200 frames collected from eight patients using a single-element $40-\mathrm{MHz}$ transducer. The same group also used a similar approach with different threshold values to detect the MA border [48]. Taki et al. [49] proposed a similar automated technique but used two different threshold values after despeckling through affine invariant anisotropic filters to detect both borders simultaneously. It has been shown that if distributions corresponding to lumen and tissue regions are well separated, simple decorrelation [50], thresholding techniques [51], [52], or unsupervised classification algorithms [53] along with morphological operations [52], [53] could lead to accurate identification of vessel borders.

Despite the simplicity of some of the presented works [47][49], [53] encouraging results were produced, especially on images acquired with low-frequency transducers $(20-30 \mathrm{MHz})$. However, in reality, the border detection problem is more complex, especially when high-frequency transducers (above $40 \mathrm{MHz}$ ) are used due to intrinsic and extrinsic image artifacts (see Section I-G) as well as local and global variations among luminal and intimal grayscale distributions, demanding for more sophisticated methods. Hence, the authors in [45] incorporated additional information about the speckle appearance, assuming a Rayleigh distribution, and modeling the expected contour with a priori knowledge using Markov processes [54] in 3-D. The final contours and parameters associated with the Rayleigh distribution were found in an iterative process employing a maximum a posteriori (MAP) estimator. The algorithm was fully automated and evaluated on 29 in vivo frames acquired with a $20-\mathrm{MHz}$ phased-array transducer. Two independent experts manually traced the vessel borders. Although large variation was observed between automated and manual lumen borders, the algorithm delivered satisfactory results. Like [45], Brusseau et al. [42] used a Rayleigh model for image brightness appearance and estimated the final contour position with a MAP estimator and a constraint on the first zero crossing of image derivatives on the borders. The presented technique was fully automated in 2-D and exploited RF lines. The possibility of ex- tension of the algorithm to 3-D was also studied by propagating 2-D contours between adjacent frames. Although ultrasound backscattered RF signals provide quantitative information on tissue microstructures [55], [56] for atherosclerotic plaque characterization, its use in border detection is somewhat limited to differentiation between blood and tissue signals and, therefore, lumen contour delineation. In addition, RF signals are not often accessible.

Cardinal et al. [57] modeled volumes of IVUS pullback images with mixtures of Rayleigh pdf corresponding to the lumen, the intima, the media, and the adventitia. They incorporated this mixture model into an extension of the fast-marching framework [58] for multiple-front propagation. They initialized the regions from manually traced lumen and media borders in several frames on longitudinal image cuts. Then, the initialized fronts propagated with different speeds proportional to each pdf. The unknown pdf parameters were estimated with the iterative expectation-maximization (EM) algorithm. They evaluated the algorithm performance with different initializations and in comparison with 3-D gradient-based fast-marching technique. Statistical analysis was then performed by comparing automated lumen and MA borders with corresponding manual ones for every other ten frames, demonstrating the robustness of the proposed methodology, obtaining small Hausdorff distance $(p<0.01)$ with different initializations. The authors in [59] also presented a 2-D semiautomated approach using a parameterization of the lumen region with a mixture of Gaussian pdfs. The curve was then deformed through the optimization of a Bayesian cost function, comprising likelihoods of grayscale intensities corresponding to blood and non-blood regions. A priori information, normalized histograms computed from manually selected regions, corresponding to blood and non-blood areas, was employed to estimate the likelihoods. Ultimately, the lumen border was detected through minimization of a cost function linearly combining the steepest descent technique and the Broyden-Fletcher-Goldfarb-Shanno (BFGS) method [60], resulting a faster convergence toward a global minimum. A set of 100 frames acquired with a $20-\mathrm{MHz}$ transducer was exploited to evaluate the technique. The accuracy was computed according to [61] and reported to be $98.28 \pm 0.49 \%, 99.43 \pm 0.29 \%$, and $95.57 \pm 1.69 \%$ for the mean, mean true negative, and mean true positive, respectively.

Although previously mentioned methods demonstrated encouraging results, the assumption of an a priori knowledge about grayscale intensities and speckle patterns through parametric distributions may not be applicable in all situations. For example, the performance of the presented methods [42], [45], [57] may be degraded if log compression is applied while Bmode images are reconstructed from RF signals. In this case, the assumption of Rayleigh distribution may not be truly valid. Alternatively, Unal et al. [46] presented an automated 2-D algorithm, implemented in the polar domain, which built a statistical shape space through principle component analysis (PCA). Once the shape space was built, an initialized contour evolved from the surface of the transducer (catheter marker) in polar coordinates by the minimization of a region-based non-parametric probabilistic energy function described in [62]. They estimated the probability distribution inside and outside the lumen using 
intensity profiles from a training dataset and delineated the lumen borders automatically. For MA border detection, they used edge information instead to evolve the curve. Primarily, this technique was developed for IVUS images acquired with a 64element phased-array $20-\mathrm{MHz}$ transducer and it demonstrated very promising results. It was later shown that the proposed algorithm provided reliable results given proper training on images acquired with single-element $40-\mathrm{MHz}$ and $45-\mathrm{MHz}$ transducers [63].

The authors in [64] suggested to combine a statistical strategy with a supervised classification approach to achieve optimal performance when detecting the MA border automatically. The core of the segmentation method consisted of three main steps. First, a restricted anisotropic diffusion (RAD) filter [30], [31] was applied for border enhancement. Second, a feature space consisting of horizontal edges, radial standard deviation, and radial cumulative mean was constructed. The former feature represented the MA border while the other two were used to differentiate between calcified and fibrotic tissue within the plaque. Fisher linear discriminant analysis [65] was then performed in order to achieve a maximum separability among the projected classes followed by Bayesian thresholding [65] in the feature space to generate two binary masks corresponding to calcified regions and the MA border. In the last step, the MA border was detected via modeling the fragmented segments in the MA border mask by computing an implicit closed representation using an anisotropic contour closing (ACC) [29] and then an explicit B-spline compact parameterization. It is also worth mentioning that thresholding of feature space was also used in [66].

\section{Multiscale Expansion-Based Techniques}

It has been shown that multiscale expansions of textured images replicate human and mammalian vision processing systems with conservation of energy in both spatial and frequency domains [67], [68]. This observation has motivated researchers to take advantage of spectral analysis [69] or spatial-frequencylocalized expansions and their generalization to 2-D as well as 3-D to discern textural patterns on backscattered IVUS images. For instance, Katouzian et al. [70] introduced the first multiscale expansion approach based on discrete wavelet packet frames (DWPF) for the automated detection of lumen borders in IVUS images acquired with a single-element $40-\mathrm{MHz}$ transducer. The proposed method relied on a 2-D overcomplete representation of IVUS images. In the first step, they decomposed images onto orthogonal Lemarie-Battle filters [71] and computed the envelope of the complex coefficients, as features. An unsupervised $K$-means clustering algorithm was then used to generate binary masks corresponding to blood and nonblood regions and the lumen border was estimated via iterative Spline interpolation among nearest detected edge sets in the radial direction. The main limitations associated with the proposed method were the presence of the guide wire, ring-down artifacts, and attenuation of signals in regions far from the transducer. Similarly, the authors in [72] also deployed discrete wavelet frames (DWF) and constructed decomposition trees using Haar filters to identify both lumen and MA borders in images collected with $20-\mathrm{MHz}$ and $40-\mathrm{MHz}$ transducers. Both techniques were able to delin- eate borders fully automatically with four decomposition levels in polar coordinates, although the detection procedures were completely different.

In a complementary study, Katouzian et al. [63], [73], [74] developed a new fully automated technique, motivated by the procedure used by interventional cardiologists to trace the lumen border in images acquired with high-frequency transducers (40 MHz up and $45 \mathrm{MHz}$ in particular). Prior to tracing, they usually go back and forth among consecutive frames to be able to visually locate the lumen contour on a single frame. By doing so, blood speckle and plaque embody, respectively, visually incoherent and coherent spatial patterns, suggesting a 3-D processing approach. IVUS subvolumes were projected onto orthogonal brushlet basis functions [75] in an overcomplete fashion [76] in polar coordinates where the strong spatial coherence in the appearance of plaque, arterial wall, and surrounding adventitia within an IVUS subvolume is better expressed. The main advantage of such a redundant representation was that the brushlet coefficients were invariant to intensity and only depended on the spatial frequency content of the IVUS signals. They proposed two different techniques to estimate the lumen border. First, they thresholded brushlet coefficients, assuming that those corresponding to plaque regions had higher magnitude and applied the iterative conditional model (ICM) segmentation framework with Markovian regularization [77] to identify the lumen borders in different classes [73]. Although the results demonstrated an efficient denoising with brushlet expansion, the ambiguity in finding the right detected lumen border remained challenging. To overcome this drawback, they later showed that the magnitudes and phases corresponding to blood and non-blood signals were well clustered in brushlet coefficients and constructed a 2.5-D magnitude-phase histograms of coefficients. Using in vitro data (circulated human mimicking blood material inside a cylindrical phantom), they demonstrated distinct histogram peaks corresponding to blood and non-blood regions. Thresholding of these peaks leads to binary masks exploited for the detection of the lumen border with the surface function active (SFA) framework [78]. In a comparison study [63], the performance of the proposed technique was comparable to the methods from [46], [49]. Furthermore, they presented dedicated approaches to remove the catheter marker and get rid of ring-down as well as guide wire artifacts.

\section{Blood Noise Reduction AND BloOd PoOL DETECTION ALGORITHMS}

To ease IVUS border detection and more specifically the luminal border, different techniques have been developed to either reduce blood speckle appearance or identify the blood pool directly. Some of the works reviewed in the previous section implemented preprocessing techniques such as median [23], [25] and anisotropic diffusion filtering [28], [29], [49], [64] prior to border detection. However, the focus of this section is on algorithms that are entirely dedicated to blood noise reduction (BNR) and blood pool detection (BPD). The term blood noise is associated with scattering from red blood cells inside the lumen. To the best of our knowledge, the authors in [79] were the first to employ signal-processing techniques in lateral direction for 
BNR in IVUS images acquired with a 20-MHz transducer. The framework exploited the Doppler shift in the blood power spectrum which could be teased out from the vessel wall spectrum centered at the zero frequency using a low-pass filter. Simulations and results driven from in vitro data demonstrated the effectiveness of the proposed technique. The rational behind most BNR and BPD algorithms [73], [80]-[83] is that blood and plaques embody incoherent and coherent textural patterns along the pullback direction. Therefore, spatiotemporal information provides discriminative features for blood speckle and blood pool. Hibi et al. [84] implemented a spatiotemporal filtering technique [83] to reduce blood noise effects. In order to validate the efficacy of the proposed method, they manually traced borders, measured lumen and plaque cross-sectional areas, and reported statistics for results before and after BNR. The authors in [80] also presented a BNR algorithm based on the fusion of anisotropically diffused filtered images [85] with temporal information and detection of the lumen borders by thresholding of edge images. A multiscale BNR algorithm was also proposed in [73] as discussed in Section II-D.

BPD has also been a subject of few studies where the presence of incoherent blood speckle patterns hindered the assessment of lumen size in IVUS images, especially for images acquired with recently developed ultrahigh-frequency transducers (40 MHz and above). O'Malley et al. [81] presented a 3-D supervised classification approach (one-class SVM) using seven spatial, temporal, and spectral features. Exploiting three in vivo datasets collected from swine coronary arteries using a 40-MHz IVUS transducer, they trained a classifier on 30 frames, and evaluated the algorithm for each dataset independently. The overall classification performance was reported as $97 \%, 82.3 \%, 2.8 \%$ and $95.3 \%, 100 \%, 4 \%$ for sensitivity, specificity, and support vector fraction, respectively, in two datasets. They concluded that the highest performance was achieved using features corresponding to speckle pattern. The authors in [86] presented a supervised technique to detect the lumen borders in longitudinal cuts of IVUS pullback images. They extracted 263 spatial, textural, and spectral features and used an Adaboost classifier for feature space dimensionality reduction and classification. Morphological operators were then deployed on classified binary images for smoothing followed by a Canny edge operator. The lumen border was detected using a parametric deformable model on detected edge points. Similarly, Katouzian et al. [82] detected blood pool and lumen borders extracting features comprising redundant brushlet coefficients along with spatial information and used a neural network as a classifier. The output of the neural network was used to reconstruct blood maps and then thresholded to estimate the lumen border with a parametric deformable model. The algorithm was evaluated using repeated randomized experiments to validate the quantification of the blood maps when compared to expert manual tracings on 258 frames collected from three patients with a single-element 45 $\mathrm{MHz}$ transducer. In order to evaluate the classification accuracy, they modified the classical correct classification rate and defined the segmentation rate $(S)$ as $(\mathrm{TP}) /(\mathrm{TP}+\mathrm{FP}+\mathrm{FN})$ for fair representation since the lumen embodied small area comparing with the whole IVUS image, causing large true negative (TN) rate. The sensitivity (SE) and specificity (SP) rates were then defined as $\mathrm{TP} /(\mathrm{TP}+\mathrm{FN})$ and $\mathrm{TN} /(\mathrm{TN}+\mathrm{FP})$, respectively, and reported to be $S=79.59 \%, \mathrm{SE}=86.80 \%, \mathrm{SP}=97.87$ for classification and $S=79.77 \%, \mathrm{SE}=89.17 \%, \mathrm{SP}=97.36 \%$ for lumen border detection results.

\section{DISCUSSION AND CONCLUSION}

The main contribution of this paper, as the first review article in the field, was to introduce state-of-the-art IVUS segmentation techniques developed over the past 20 years. Avoiding direct comparison, we rather categorized algorithms into three groups: direct detection of the MA or the lumen borders, classification of luminal and blood pool areas, and blood speckle noise reduction (BNR) methods. Techniques belonging to the first group (direct detection) are usually preferred as they offer instant measurements of lumen and plaque areas, which are required prior to tissue characterization or balloon angioplasty and stent implantation. Although all reviewed algorithms bring specific advantages, they have been only partially successful in clinical setting. For very precise measurements, e.g., plaque progression/regression studies during pharmacological trials, most of the research laboratories still rely on manually traced borders.

To make these methods usable in clinical setting, an automated adaptation of the algorithms to morphological and pathological textural changes within pullback series of images needs to be provided. Indeed, most of the validation datasets used in the literature comprise frames from distinct parts of pullback series, which do not reflect the needs during catheterization procedures. Across hundreds to thousands of frames, a pullback series encounters merging of side branches, enlargement of calcified tissue arcs (e.g., larger than $120^{\circ}$ ), and variation of ring-down artifact patterns. Despite the fact that aforementioned factors play important roles on segmentation results, only few works [46], [49], [63] address these issues implicity. Therefore, reporting statistics on temporally diverse dataset is of low importance and does not guarantee the claimed reliability or the usability of the algorithm for real-time application in a catheterization laboratory.

Second, in most studies, the grayscale distributions corresponding to blood and non-blood regions are assumed to be separable. This is a reasonable assumption for images acquired with low-frequency transducers $(20-30 \mathrm{MHz})$, and the authors in [63] have shown that, in such a situation, a thresholding-based technique with empirically tuned values [49] outperforms more sophisticated algorithms [46], [74]. However, this assumption is no longer valid for images acquired with transducers $(40 \mathrm{MHz}$ and above). We illustrate this point in Fig. 8 which shows empirical intensity distributions derived from images acquired with 20-, 40-, and 45-MHz transducers. As we can see, the separation between blood and non-blood intensities vanishes when the transducer center frequency increases from 20 to $45 \mathrm{MHz}$. As more scattering from red blood cells emerges with the increase in the center frequency of the transducer, visual detection of luminal borders becomes impossible without exploiting the longitudinal incoherence of the blood patterns.

Third, the literature has reported different metrics to compare the segmentation results to manually traced borders, making direct comparisons very difficult. As we can see in Table I, linear 


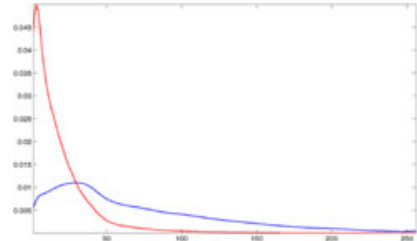

(a)

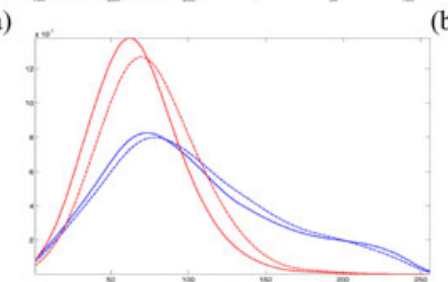

(c)

Fig. 8 Probability distribution of blood (red) and nonblood (blue) regions derived from images acquired with (a) $20-\mathrm{MHz}$, (b) $40-\mathrm{MHz}$, and (c) $45-\mathrm{MHz}$ transducers. The probability distributions were computed from two datasets (solid and dashed lines).

regression is the most common analysis, which seems necessary but is insufficient. On the other hand, clinicians usually prefer Bland-Altman graphs to visualize potential bias in errors with respect to vessel diameters. The inter- and intra-observer manual variability enables us to quantify the consistency and reproducibility of quantitative measurements made by different observers tracing the borders. For example, the average interand intra-observer correlation coefficients reported in [18] and [23] for lumen and MA borders are $r_{\text {inter }}=0.962, r_{\text {intra }}=0.988$ and $r_{\text {inter }}=0.965, r_{\text {intra }}=0.999$, respectively. As we can see, the visual tracing of MA borders is also relatively easier than lumen. The same conclusion can also be made where lower interand intra-observer $(4.5 \pm 3.3 \%$ and $3.4 \pm 3.4 \%)$ variability for MA borders in comparison with lumen borders $(10.5 \pm 11.0 \%$ and $8.3 \pm 8.9 \%$ ) was reported in [35].

Finally, there are still no common datasets freely distributed on which different algorithms could be compared. A joint effort to develop such a database, as it has been done in the past for coronary segmentation on CT or carotid segmentation for example, is urgently needed.

In conclusion, to drive automated quantification tools to the clinics, more standardization in the validation process, performance metrics, and construction of globally accessible databases consisting of pullbacks of images that imitate all possible scenarios in real catheterization procedures, acquired with different transducer frequencies, are required.

\section{REFERENCES}

[1] E. J. Topol and S. E. Nissen, "Our preoccupation with coronary lumenology. The dissociation between clinical and angiographic findings in ischemic heart disease," Circulation, vol. 92, pp. 2333-2342, 1995.

[2] F. M. Sones and E. K. Shirey, "Cine coronary arteriography," Mod. Conc. Cardiovasc. Dis., vol. 31, pp. 735-738, 1962.

[3] W. C. Little, "Angiographic assessment of the culprit coronary artery lesion before acute myocardial infarction," Amer. J. Cardiol., vol. 66, pp. 44G-47G, 1990.

[4] J. E. Muller and G. H. Tofler, "Triggering and hourly variation of onset of arterial thrombosis," Ann. Epidemiol., vol. 2, pp. 393-405, 1992.

[5] E. Falk, "Why do plaques rupture?," Circulation, vol. 86, pp. III30-III42, 1992.
[6] P. Libby, "Molecular bases of the acute coronary syndromes," Circulation, vol. 91, pp. 2844-2850, 1995.

[7] R. Virmani, A. P. Burke, F. D. Kolodgie, and A. Farb, "Pathology of the thin-cap fibroatheroma: A type of vulnerable plaque," J. Inteven. Cardiol., vol. 16 , no. 3, pp. 267-272, 2003.

[8] I. K. Jang, B. E. Bouma, D. H. Kang, S. J. Park, S. W. Park, K. B. Seung, K. B. Choi, M. Shishkov, K. Schlendrof, E. Pomerantsev, S. L. Houser, H. T. Aretz, and G. J. Tearney, "Visualization of coronary atherosclerotic plaques in patients using optical coherence tomography: Comparison with intravascular ultrasound," J. Amer. Coll. Cardiol., vol. 39, pp. 604-609, 2002.

[9] J. D. Caplan, S. Waxman, R. W. Nesto, and J. E. Muller, "Near-infrared spectroscopy for the detection of vulnerable coronary artery plaques," $J$. Amer. Coll. Cardiol., vol. 47, pp. C92-C96, 2006.

[10] G. W. Stone, A. Maehara, A. J. Lansky, B. de Bruyne, E. Cristea, G. S. Mintz, R. Mehran, J. McPherson, N. Farhat, S. P. Marso, H. Parise, B. Templin, R. White, Z. Zhang, and P. W. Serruys, "A prospective naturalhistory study of coronary atherosclerosis," N. Engl.J. Med., no. 364, pp. 226-235, 2011.

[11] ACC/AHA/SCAI Writing Committee, "American College of Cardiology/ American Heart Association Task Force on practice guidelines," J. Amer. Coll. Cardiol., vol. 47, no. 1, pp. e1-e121, 2006.

[12] A. V. Oppenheim and R. W. Schafer, Discrete-Time Signal Processing, 2nd ed. Englewood Cliffs, NJ: Prentice-Hall, 1998.

[13] D. M. Herrington, T. Johnson, P. Santago, and W. E. Sydner, "Semiautomated boundary detection for intravascular ultrasound," in Proc. Comput. Cardiol., 1992, vol. 192, pp. 103-106.

[14] M. Sonka, X. Zhang, M. Siebes, M. S. Bissing, S. C. DeJong, S. M. Collins, and C. R. McKay, "Segmentation of intravascular ultrasound images: A knowledge-based approach," IEEE Trans. Med. Imag., vol. 14, no. 4, pp. 719-732, Dec. 1995.

[15] M. Sonka, V. Hlavac, and R. Boyle, Image Processing, Analysis, and Machine Vision. $\quad$ London, U.K.: Chapman \& Hall, 1993.

[16] N. J. Nilsson, Problem Solving Methods in Artificial Intelligence. New York: McGraw Hill, 1971.

[17] R. Downe, A. Wahle, T. Kovarnik, H. Skalicka, J. Lopez, J. Horak, and M. Sonka, "Segmentation of intravascular ultrasound images using graph search and a novel cost function," in Proc. Medical Image Computing and Computer Assisted Intervention Workshop on Computer Vision for Intravascular and Intracardiac Imaging, New York, 2008, pp. 71-79.

[18] C. von Birgelen, C. Mario, W. Li, J. Schuurbiers, C. Slager, P. de Feyter, P. Serruys, and J. Roelandt, "Morphometric analysis in three-dimensional intracoronary ultrasound: An in vitro and in vivo study using a novel system for the contour detection of lumen and plaque," Amer. Heart J., vol. 132 , no. 3, pp. 516-527, 1996.

[19] W. Li, C. von Birgelen, C. Di Mario, E. Boersma, E. J. Gussenhoven, N. Van der Putten, and N. Bom, "Semi-automatic detection for volumetric quantification of intracoronary ultrasound," in Proc. Comput. Cardiology, Bethesda, MD, 1994, pp. 277-280.

[20] C. von Birgelen, A. van der Lugt, A. Nicosia, G. S. Mintz, E. J. Gussenhoven, E. de Vrey, M. T. Mallus, J. R.T.C. Roelandt, P. W. Serruys, and P. J. de Feyter, "Computerized assessment of coronary lumen and atherosclerotic plaque dimensions in three-dimensional intravascular ultrasound correlated with histomorphometry," Amer. J. Cardiol., vol. 7, pp. 1202-1209, 1996.

[21] C. von Birgelen, G. S. Mintz, A. Nicosia, D. P. Foley, W. J. van der Giessen, N. Bruining, S. G. Airiian, J. R. T. C. Roelandt, P. J. de Feyter, and P. W. Serruys, "Electrocardiogram-gated intravascular ultrasound image acquisition after coronary stent deployment facilitates on-line threedimensional reconstruction and automated lumen quantification," J. Amer. Coll. Cardiol., vol. 30, pp. 436-443, 1997.

[22] J. Dijkstra, G. Koning, J. C. Tuinenburg, P. V. Oemrawsingh, and J. H. G. Reiber, "Automatic border detection in intravascular ultrasound images for quantitative measurements of the vessel, lumen and stent parameters," Comp. in Cardiol, pp. 25-28, 2001.

[23] D. Meier, R. Cothren, D. Vince, and J. Cornhill, "Automated morphometry of coronary arteries with digital image analysis of intravascular ultrasound," Amer. Heart J, vol. 133, no. 6, pp. 681-690, 1997.

[24] Z. Luo, Y. Wang, and W. Wang, "Estimating coronary artery lumenarea with optimization-based contour detection," IEEE Trans. Med. Imag., vol. 22, no. 4, pp. 564-566, Apr. 2003.

[25] M. Schmauder, C. M. Gross, M. Firle, J. Waigand, and R. Orglmeister, "Automated detection of endovascular contours from intravascular ultrasound image sequence using a simulated annealing approach. Book of abstracts," presented at the 8th Mediterranean Conf. Medical and Biological Engineering and Computing, Lemesos, Cyprus, Jun. 14-17, 1998. 
[26] M. Kass, A. Witkin, and D. Terzopoulos, "Snakes: Active contour models," Int. J. Comput. Vision, pp. 321-331, 1988.

[27] D. J. Williams and M. Shah, "A fast algorithm for active contours and curvature estimation," CVGIP-Image Understand., vol. 55, no. 1, pp. 14 26, 1997.

[28] A. Hernandez, D. Gil, P. Radeva, and E. Nofrerias, "Anisotropic processing of image structures for adventitia detection in IVUS images," in Proc. Computers in Cardiology, 2004, vol. 31, pp. 229-232.

[29] D. Gil, P. Radeva, and J. Mauri, "IVUS segmentation via a regularized curvature flow," presented at the XX Congreso Anual de la Sociedad Española de Ingeniería Biomédica, Zaragoza, Nov. 27-29, 2002.

[30] D. Gil, "Geometric differential operators for shape modeling,", Ph.D. dissertation, Univ. Autonoma de Barcelona, Barcelona, Spain, 2004.

[31] D. Gil and P. Radeva, "Extending anisotropic operators to recover smooth shapes," Comput. Vis. Imag. Under., vol. 99, pp. 110-125, 2005.

[32] D. Gil and P. Radeva, "A regularized curvature flow designed for a selective shape restoration," IEEE Tran. Imag. Proc., vol. 13, no. 11, pp. 14441458, Nov. 2004.

[33] M. Plissiti, D. Fotidadis, L. Michalis, and G. Bozios, "An automated method for lumen and media-adventitia border detection in a sequence of IVUS frames," IEEE Trans. Info. Tech. Biomed., vol. 8, no. 2, pp. 131141, Jun. 2004.

[34] Y. Zhu and H. Yan, "Computerized tumor boundary detection using a Hopfield neural network," IEEE Trans. Med. Imag., vol. 16, no. 1, pp. 5567, Feb. 1997

[35] G. Kovalski, R. Beyar, R. Shofti, and H. Azhari, "Three-dimensional automatic quantitative analysis of intravascular ultrasound images," $\mathrm{Ul}$ trasound Med. Biol., vol. 26, no. 4, pp. 527-537, 2000.

[36] L. D. Cohen, "On active contour models and balloons," CVGIP: Image Understand., vol. 53, pp. 211-218, 1991.

[37] R. Shekhar, R. M. Cothren, D. G. Vince, S. Chandra, J. D. Thomas, and J. F. Cornhill, "Three-dimensional segmentation of luminal and adventitial borders is serial intravascular ultrasound images," Computer. Med. Imag. Graphics, vol. 23, pp. 299-309, 1999.

[38] S. Lobregt and M. A. Viergever, "Discrete dynamic contour model," IEEE Trans. Med. Imag., vol. 14, no. 1, pp. 12-24, Mar. 1995.

[39] J. D. Klingensmith, R. Shekhar, and D. G. Vince, "Evaluation of threedimensional segmentation algorithms for the identification of luminal and medial-adventitia borders in intravascular ultrasound images," IEEE. Tran. Med. Imag., vol. 19, no. 10, pp. 996-1011, Oct. 2000.

[40] C. Haas, H. Ermert, S. Holt, P. Grewe, A. Machraoui, and J. Barmeyer, "Segmentation of 3D intravascular ultrasonic images based on a random field model," Ultrasound Med. Biol., vol. 26, no. 2, pp. 297-306, 2000.

[41] H. Zhu, Y. Liang, and M. Friedman, "IVUS image segmentation based on contrast," in Proc. SPIE, 2002, vol. 4684, pp. 1727-1733.

[42] E. Brusseau, C. deKorte, F. Mastik, J. Schaar, and A. van der Steen, "Fully automatic luminal contour segmentation in intracoronary ultrasound imaging-A statistical approach," IEEE Trans. Med.Imag., vol. 23, no. 5 , pp. 554-566, May 2004.

[43] M. H. Cardinal, J. Meunier, G. Soulez, R. Maurice, E. Therasse, and G. Cloutier, "Automatic 3D segmentation of intravascular ultrasound images using region and contour information," in Medical Image Computing and Computer-Assisted Intervention (Lecture Notes in Computer Science, vol. 3749), 2005, pp. 319-326.

[44] P. Oriol and P. Radeva, "Lumen detection in IVUS images using snakes in a statistical framework," presented at the XX Congreso Anual de la Sociedad Española de Ingeniería Biomédica, Zaragoza, Nov. 27-29, 2002.

[45] C. Haas, H. Ermert, S. Holt, P. Grewe, A. Machraoui, and J. Barmeyer, "Segmentation of 3D intravascular ultrasonic images based on a random field model," Ultrasound Med. Biol., vol. 26, no. 2, pp. 297-306, 2000.

[46] G. Unal, S. Bucher, S. Carlier, G. Slabaugh, T. Fang, and K. Tanaka, "Shape-driven segmentation of the arterial wall in intravascular ultrasound images," IEEE Trans. Info. Tech. Biomed., vol. 12, no. 3, pp. 335-347, May 2008.

[47] D. Gil, P. Radeva, J. Saludes, and J. Mauri, "Automatic segmentation of artery wall in coronary IVUS images: A probabilistic approach," in Proc. Int. Conf. Pattern Recog., 2000, pp. 4352-4355.

[48] D. Gil, P. Radeva, and J. Saludes, "Segmentation of artery wall in coronary IVUS images: A probabilistic approach," in Proc. 15th Int. Conf. Pattern Recog., Barcelona, Spain, 2000, vol. 4, pp. 352-355.

[49] A. Taki, Z. Najafi, A. Roodaki, S. K. Setarehdan, R. A. Zoroofi, A. Konig, and N. Navab, "Automatic segmentation of calcified plaques and vessel borders in IVUS images," Int. J. CARS, vol. 3, pp. 347-354, 2008.

[50] W. Li, A. F. van der Steen, C. T. Lancee, J. Honkoop, E. J. Gussenhoven, and N. Bom, "Temporal correlation of blood scattering signals in vivo from radiofrequency intravascular ultrasound," Ultrasound Med. Biol., vol. 22, no. 5, pp. 583-590, 1996.

[51] J. Vandenberg, G. Liersch, H. Hanna, and J. Cameron, "Fully automated media and lumen boundary detection in intravascular ultrasound images," in Proc. IEEE Southwest Symp. Image Anal. Interpretation, 1996, pp. 7175

[52] C. J. Bouma, W. J. Niessen, K. J. Zuiderveld, E. J. Gussenhoven, and M. A. Viergever, "Evaluation of segmentation algorithms for intravascular ultrasound images," in Proc. 4th Int. Conf. Visualization in Biomedical Computing, 1996, pp. 203-212.

[53] E. Dos Santos, M. Yoshizawa, A. Tanaka, Y. Saijo, and T. Iwamoto, "Detection of luminal contour using fuzzy clustering and mathematical morphology in intravascular ultrasound images," in Proc. Int. Conf. IEEE Eng. Med. Biol., 2005, pp. 3471-3474.

[54] J. Besag, "Spatial interaction and the statistical analysis of lattice systems," J. Ro. Stat. Soc. B., vol. 36, pp. 192-236, 1974.

[55] L. Fellingham and F. Sommer, "Ultrasonic characterization of tissue structure in the in vivo human liver and spleen," IEEE Trans. Sonics Ultrason., vol. SU-31, no. 4, pp. 418-428, Jul. 1984.

[56] L. Landini and L. Verrazzani, "Spectral characterization of tissue microstructure by ultrasound: A stochastic approach," IEEE Trans. Ultrason. Ferroelectr. Freq. Control, vol. 37, no. 5, pp. 448-456, Sep. 1990.

[57] M. R. Cardinal, J. Meunier, G. Soulez, R. L. Maurice, E. Therasse, and G. Cloutier, "Intravascular ultrasound image segmentation: A threedimensional fast-marching method based on gray level distribution," IEEE. Trans. Med. Imag., vol. 25, no. 5, pp. 590-601, May 2006.

[58] E. Sifakis, C. Garcia, and G. Tziritas, "Bayesian level sets for image segmentation," J. Vis. Commun. Image Rep., vol. 13, pp. 44-64, 2002.

[59] E. Mendizabal-Ruiz, M. Rivera, and I. Kakadiaris, "A probabilistic segmentation method for the identification of luminal borders in intravascular ultrasound images," in Proc. IEEE Comput. Soc. Conf. Comput. Vision Pattern Recog., 2008, pp. 1-8.

[60] J. Nocedal and S. Wright, Numerical Optimization. New York: Springer, 1999.

[61] J. Udupa, Y. Jin, C. Imielinska, A. Laine, W. Shen, and S. Heymsfield, "Segmentation and evaluation of adipose tissue from whole body MRI scans," in Medical Image Computing and Computer-Assisted Intervention, LNCS 28, 2003, pp. 635-642.

[62] N. Paragios and R. Deriche, "Geodesic active regions and level set methods for supervised texture segmentation," Int.J. Comput. Vis., vol. 46, no. 3 , pp. 223-247, 2002.

[63] A. Katouzian, "Quantifying atherosclerosis: IVUS imaging for lumen border detection and plaque characterization," Ph.D. dissertation, Columbia Univ., NY, 2011.

[64] D. Gil, A. Hernandez, O. Rodriguez, J. Mauri, and P. Radeva, "Statistical strategy for anisotropic adventitia modeling in IVUS," IEEE Trans. Med. Imag., vol. 25, no. 6, pp. 768-778, Jun. 2006.

[65] R. Duda and P. Hart, Pattern Classification. New York: WileyInterscience, 2001

[66] A. Mojsilovic, M. Popovic, N. Amodaj, R. Babic, and M. Ostojic, "Automatic segmentation of intravascular ultrasound images: A texture-based approach," Ann. Biomed. Eng., vol. 25, pp. 1059-1071, 1997.

[67] R. De Valois and K. De Valois, Spatial Vision. New York, NY: Oxford Univ. Press, 1988.

[68] J. Beck, A. Sutter, and R. Ivry, "Spatial frequency channels and perceptual grouping in texture segregation," Comput. Vision, Graph., Image Proc., vol. 37, pp. 299-325, 1987.

[69] J. Klingensmith, A. Nair, B. Kuban, and D. Vince, "Segmentation of threedimensional intravascular ultrasound images using spectral analysis and a dual active surface model," in Proc. IEEE Int. Ultrason. Symp., Sep. 2004, vol. 2, pp. 1765-1768.

[70] A. Katouzian, B. Baseri, E. E. Konofagou, and A. F. Laine, "Automatic detection of blood versus non-blood regions on intravascular ultrasound (IVUS) images using wavelet packet signatures," Proc. SPIE, vol. 6920, pp. $69200 \mathrm{H} 1-8$, Feb. 2008 .

[71] S. G. Mallat, "A theory of multiresolution signal decomposition: The wavelet representation," IEEE Trans. Pat. Anal. Mach. Intell., vol. 11, no. 7, pp. 674-693, Jul. 1989

[72] M. Papadogiorgaki, V. Mezaris, Y. Chatzizisis, G. Giannoglou, and I. Kompatsiaris, "Image analysis techniques for automated IVUS contour detection," Ultrasound Med. Biol., vol. 34, no. 9, pp. 1482-1498, 2008.

[73] A. Katouzian, E. Angelini, A. Lorsakul, B. Sturm, and A. Laine, "Lumen border detection of intravascular ultrasound via denoising of directional wavelet representations," in Proc. Functional Imaging and Modeling of the Heart, 2009, pp. 104-113. 
[74] A. Katouzian, E. D. Angelini, B. Sturm, and A. F. Laine, "Automatic detection of luminal borders in IVUS images by magnitude-phase histograms of complex brushlet coefficients," in Proc. IEEE Eng. Med. Biol. Soc., Buenos Aires, Argentina, 2010, pp. 3073-3076.

[75] F. Meyer and R. R. Coifman, "Brushlets: A tool for directional image analysis and image compression," Appl. Comput. Harmonic Anal., vol. 4, pp. 147-187, 1997.

[76] E. Angelini, A. Laine, S. Takuma, J. Holmes, and S. Homma, "LV volume quantification via spatio-temporal analysis of real-time 3D echocardiography," IEEE Trans. Med. Imag., vol. 20, no. 6, pp. 457-469, Jun. 2001.

[77] J. Besag, "On the statistical analysis of dirty pictures," J. Roy. Stat. Soc., Series B, vol. 48, no. 3, pp. 259-302, 1986.

[78] Q. Duan, E. D. Angelini, and A. F. Laine, "Surface function actives," $J$. Vis. Commun. Image R., vol. 20, pp. 478-490, 2009.

[79] A. Gronningsaeter, B. A. J. Angelsen, A. Gresli, H. G. Torp, and D. T. Linker, "Blood noise reduction in intravascular ultrasound images," IEEE Trans. Ultrason. Ferroelectr. Freq. Control, vol. 42, no. 2, pp. 200 209, Mar. 1995.

[80] S. Balocco, O. Basset, C. Cachard, and P. Delachartre, "Spatial aniosotropic diffusion and local time correlation applied to segmentation of vessels in ultrasound image sequences," in Proc. IEEE Symp. Ultrasonics, Oct. 2003, vol. 2, pp. 1549-1552.

[81] S. M. O'Malley, M. Naghavi, and I. A. Kakadiaris, "One-class acoustic characterization applied to blood detection in IVUS," in Medical Image Computing and Computer-Assisted Intervention (Lecture Notes in Computer Science, vol. 4791), 2007, pp. 202-209.

[82] A. Katouzian, M. A. Selver, E. Angelini, B. Sturm, and A. Laine, "Classification of blood regions in IVUS images using three dimensional brushletderived features," in Proc. Int. Conf. IEEE Eng. Med. and Biol. Soc., 2009, pp. 471-474.

[83] X. Zhang and T. J. Teo "Method and apparatus for spatial and temporal filtering of intravascular ultrasonic image data," World Intellectual Property Organization Patent, International Publication Number WO 00/07035, 2000.

[84] K. Hibi, A. Takagi, X. Zhang, T. T. Teo, H. N. Boneau, P. G. Yock, and P. J. Fitzgerald, "Feasibility of a novel blood reduction algorithm to enhance reproducibility of ultra-high-frequency intravascular ultrasound images," Circulation, vol. 102, no. 14, pp. 1657-1663, 2000.

[85] Y. Yu and S. T. Acton, "Speckle reducing anisotropic diffusion," IEEE Trans. Image Process., vol. 11, no. 11, pp. 1260-1271, Nov. 2002.

[86] D. Rotger, P. Radeva, E. F. Nofrerias, and J. Mauri, "Blood detection in IVUS images for 3D volume of lumen changes measurement due to different drugs administration," in Computer Analysis of Image and Patterns (Lecture Notes in Computer Science vol. 4673), 2007, pp. 285-292.

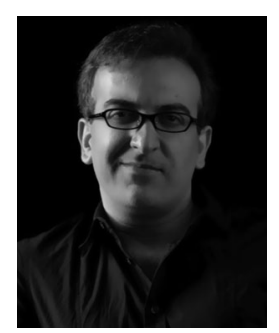

Amin Katouzian received the Ph.D. degree from Columbia University, New York, in 2011.

$\mathrm{He}$ is currently a Senior Research Scientist at the Chair for Computer Aided Medical Procedures, Technical University of Munich, Munich, Germany, as well as at Heffner Biomedical Imaging Lab, Columbia University. His main research interests include computer vision for medical applications, multidimensional analysis and cue extraction, pattern recognition, and medical image analysis.

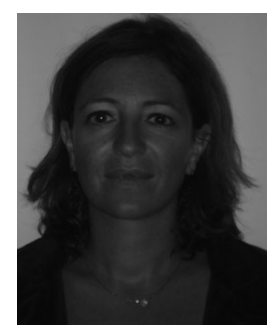

Elsa D. Angelini (M'98) received the B.Sc. degree from the Ecole Centrale de Nantes, Nantes, France, in 1996, and the M.Sc. and Ph.D. degrees in biomedical engineering from Columbia University, New York, in 1998 and 2003, respectively.

She is currently an Associate Professor of Computer Science at the Institut Telecom, TelecomParisTech, Paris, France, and a Visiting Professor in the Department of Biomedical Engineering, Columbia University. Her research focuses on image processing for multidimensional biomedical imaging, including applications such as denoising, enhancement, segmentation, and modeling.

Dr. Angelini is an Associate Editor for the IEEE TRANSACTIONS ON BIOMEDICAL ENGINEERING.

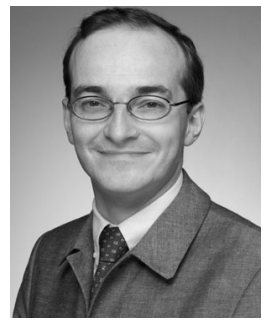

Stéphane G. Carlier received the MD degree from the Université Libre de Bruxelles, Brussel, Belgium, in 1996 and the Ph.D. degree in biomedical engineering from Erasmus University, Rotterdam, The Netherlands, in 2001.

$\mathrm{He}$ is currently an Interventional Cardiologist at the University Hospital (UZ), Brussel, and at St Maria Hospital, Halle, Belgium. He was an Assistant Professor of Clinical Medicine at Columbia University, New York, and also the Director of the Intravascular Imaging and Physiology Corelab, Cardiovascular Research Foundation. He serves on several reviewing boards of medical and bioengineering journals. His research interests include intravascular imaging, cardiac and vascular dynamics, physiology, and signal processing.

Dr. Carlier is a member of the European Association of Percutaneous Cardiovascular Interventions of the European Society of Cardiology.

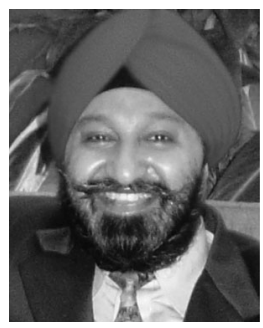

Jasjit S. Suri received the Ph.D. degree in electrical engineering (in conjunction with Cardiology Division, School of Medicine) from the University of Washington, Seattle, and the MBA degree from the Weatherhead School of Management (in conjunction with the Department of Biomedical Engineering), Case Western Reserve University, Cleveland, $\mathrm{OH}$.

$\mathrm{He}$ is an innovator, visionary, scientist, and an internationally known world leader, and has spent more than 25 years in the field of biomedical engineering/sciences and its management. He has written more than 350 peer-reviewed technical publications. He has championed the field imaging sciences. He is a committee member of several journals and companies.

Dr. Suri was crowned with the President's Gold medal in 1980 and a Fellow of the American Institute of Medical and Biological Engineering, awarded by National Academy of Sciences, Washington, DC, in 2004. He has been the Chairman of the IEEE Denver section and has won more than 50 awards during his career.

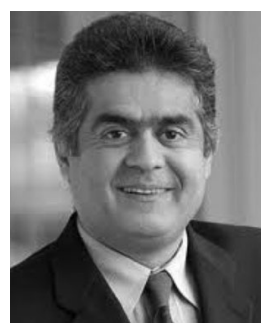

Nassir Navab received the Ph.D. degree from the Institut National de Recherche en Informatique et en Automatique (INRIA), Rocquencourt, France.

$\mathrm{He}$ is currently a Full Professor and Director of the Institute for Computer Aided Medical Procedures (CAMP), Technical University of Munich, Munich, Germany, with a secondary faculty appointment at its Medical School. He is also acting as Chief Scientific Officer for SurgicEye, Munich. In November 2006, he was elected as a member of board of directors of MICCAI society. He is the author of hundreds of peer reviewed scientific papers and over 60 U.S. and international patents.

Dr. Navab has served on the Steering Committee of the IEEE Symposium on Mixed and Augmented Reality between 2001 and 2008. He has received the Siemens Inventor of the Year Award in 2001, and the SMIT Technology Award in 2010. He has also co-authored many awarded papers in prestigious conferences including the IEEE FG 2011, MICCAI 2007, 2009 and 2010, IEEE ISMAR 2005, VOEC-ICCV 2009, AMDO 2009, BMVC 2009, and IPMI 2007.

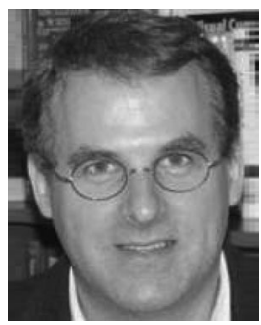

Andrew F. Laine (F'10) received the D.Sc. degree from the School of Engineering and Applied Science in Computer Science, Washington University, St. Louis, in 1989

He is currently the Chair of the Department of Biomedical Engineering and the Director of Heffner Biomedical Imaging Lab, Columbia University, New York. His main research interests include quantitative image analysis, cardiac functional imaging, ultrasound and MRI, retinal imaging, intravascular imaging, and biosignal processing.

Dr. Laine has been the Vice President of publications for the IEEE EMBS since 2008. He is a Fellow of the American Institute of Medical and Biological Engineering (AIMBE). 